\title{
A Kernel-Oriented Algorithm for Transmission Expansion Planning
}

\author{
Javier Contreras, Member, IEEE and Felix F. Wu, Fellow, IEEE
}

\begin{abstract}
With deregulation sweeping all over electrical systems around the world, transmission planning has undergone dramatic changes during this decade. Centralized cost allocation methods have become obsolete and new procedures are needed to deal with intelligent and self-sufficient players. In this paper we study the allocation of transmission costs in a decentralized manner. For this purpose we have developed a multi-agent system that is based on a well known cooperative game theory procedure, the kernel. Using our approach, the agents are able to form kernel-stable coalitions and the cost allocation procedure is performed at every step of the kernel- algorithm. A six bus example and an IEEE 24 bus case illustrate our model.
\end{abstract}

Index Terms-Cooperative game theory, kernel, multi-agent systems, transmission planning.

\section{INTRODUCTION}

$\mathbf{T}$ RANSMISSION planning has been traditionally centralized until deregulation has been adopted by different countries around the world.

Historically, a seminal work by Garver formulated the transmission expansion problem in mathematical notation in the early seventies [1]. During this decade, mixed-integer programming techniques using Benders decomposition [2]-[4], simulated annealing [5], genetic algorithms [6], and artificial neural networks [7] are among the latest contributions to the field.

However, in the new deregulated environment, new features related to transmission expansion and also connected to transmission pricing have been pointed out by several authors [8]. In particular, economies of scale and synergies in the expansion may become crucial in decentralized planning models.

On the other hand, not only expansion plans but also fair ways to split lines' usage costs have been recently studied [9]-[11]. The MW-mile method and other embedded cost methods are currently under heavy modifications in order to account for wheeling transactions among the users of the network.

Game theory has also been used to model the sharing of costs among the users of new transmission lines. Gately modeled a centralized cooperative game theory framework where the investments were shared according to the Shapley values of the

Manuscript received September 23, 1999.

J. Contreras is with the E.T.S. de Ingenieros Industriales, Universidad de Castilla-La Mancha, 13071 Ciudad Real, Spain (e-mail: javier@ind-cr.uclm.es).

F. F. Wu is with the Department of Electrical and Electronic Engineering,

University of Hong Kong, Hong Kong (e-mail: ffwu@eee.hku.hk).

Publisher Item Identifier S 0885-8950(00)10382-7. game of expansion [12]. However, this approach was not applicable to an environment where the players take their decisions looking for their own benefit only.

Distributed Artificial Intelligence has proved a valuable tool to aid game theory coping with multi-agent decision systems in decentralized environments. The combination of both techniques has been already applied to solve transmission expansion simple scenarios [13], [14]. The use of bilateral Shapley values and a cost allocation technique based on a backward induction method has been successful with simple examples.

This paper is an extension of the original transmission expansion problem studied in [13] that explores a new methodology: the kernel approach. The kernel is a concept from cooperative game theory that splits a common resource among the players in terms of the "strength" of the members of the coalition. In particular, for the transmission expansion game, it rewards the members that are less costly to the expansion of the system.

The structure of the paper is as follows. Section II covers the mathematical model of the transmission planning problem. Section III describes multi-agent settings and their connection to game theory. Section IV analyzes the kernel approach and the cost allocation procedure. Section V shows the results from a 6 bus example and an IEEE 24 bus case. Finally, Section VI draws several conclusions.

\section{The Transmission Planning Problem}

A simplified formulation of the transmission expansion problem can be expressed as follows [15]:

$$
\min \frac{1}{2} \sum_{j=1}^{M} c_{j} P_{j}^{2}
$$

subject to

$$
\mathbf{B} \Theta+\mathbf{K}^{T} \mathbf{P}_{D}=\mathbf{P}
$$

$$
\left|\mathbf{B}_{L} \mathbf{A} \Theta\right| \leq \overline{\mathbf{P}}_{L}
$$

where

$c_{j} \quad$ is the cost of adding line $j$ to the network,

$P_{j} \quad$ is the active power (in p.u.) flowing through the added line $j$, i.e. the $j$ th element of $\mathbf{P}_{D}$, and

$\mathbf{P}_{D}$ is the flow vector for the possible new lines.

$M$ is the number of possible new lines, $\mathbf{B}$ is the matrix whose elements are the imaginary parts of the nodal admittance matrix of the existing network, $\Theta$ is the phase angle vector, $\mathbf{K}^{T}$ is the 
transpose of the node-branch connection matrix, $\mathbf{P}$ is the nodal injection power for the overall network, $\mathbf{B}_{L}$ is a diagonal matrix whose elements are the branch admittances, $\overline{\mathbf{P}}_{L}$ is the branch active power vector, and $\mathbf{A}$ is the network incidence matrix.

\section{Multi-Agent Setting And Game Theory}

The main advantage of a centralized planning system is its simplicity. Using a central planner, we do not need a synchronized algorithm to exchange information amongst coordinator and agents, neither among the agents themselves. A multi-agent system with decentralized decision making is a more complex system. Then, why using such a complex system?

In real life, an agent is just an autonomous entity that makes decisions according to her own intelligence and also depending on the actions of other players. A system with many autonomous decision makers is called a multi-agent system. In transmission planning, there are many agents or players that can make decision about expanding the network or not. In particular, generators, loads and transmission line owners. It is not easy to determine how these agents can obtain the maximum individual benefit from expanding the line, and if it is better or not to form coalitions to gain more.

To solve this problem, this paper proposes a multi-agent system that is driven by coalitional agreements based on cooperative game theory. Cooperative game theory is a branch of game theory that deals with players that are willing to join other players in order to obtain more benefit. Indeed this is the case of transmission planning, where bigger coalitions need less lines to expand the network, since the overall reliability is improved if there are more players. However, this decentralized and distributed decision making process needs a coordinator to ensure that the flow of information is synchronized. The next section presents the mathematical formulation that the agents use to build coalitions, based on a solution formula called the kernel, within a multi-agent framework.

\section{CoAlition Formation And Cost Allocation: A KERNEL APPROACH}

The kernel [16] is a cooperative game theory solution concept in which the coalitional configurations are stable in the sense that there is an equilibrium between pairs of individual agents which are in the same coalition. Two agents $A, B$ in a coalition $C$ are in equilibrium if they cannot outweight one another from $C$, their common coalition. Agent $A$ can outweight $B$ if $A$ is stronger than $B$, where strength refers to the potential of agent $A$ to successfully claim a part of the payoff of agent $B$.

In each stage of the coalition formation process, the agents are in a coalitional configuration. That is, the agents are arranged in a set of coalitions $\mathcal{C}=\left\{C_{i}\right\}$. During the coalition formation process agents can use the kernel solution concept to object to the payoff distribution that is attached to their coalitional configuration. The objections that agents can make are based on the excess concept. The relevant definitions are recalled now.
Excess: The excess ${ }^{1}$ of a coalition $C$ with respect to a coalitional configuration $C$ is defined as

$$
e(C)=V(C)-\sum_{A_{i} \in C} u^{i}
$$

where $u^{i}$ is the payoff of agent $A_{i}$ and $V(C)$ is the coalitional value of coalition $C$. The number of excesses is an important property of the kernel solution concept. Agents use the excesses as a measure of their relative strengths. Since a higher excess correlates with more strength, rational agents must search for the highest excess they have. The maximum is defined by the surplus.

Surplus and Outweight: The maximum surplus $S_{A B}$ of agent $\mathrm{A}$ over agent $\mathrm{B}$ with respect to a coalitional configuration is defined by

$$
S_{A B}=\max _{C \mid A \in C, B \notin C} e(C)
$$

where $e(C)$ are the excesses of all the coalitions that include $A$ and exclude $B$, and the coalitions $C$ are not in the current coalitional configuration. Agent $A$ outweights agent $B$ if $S_{A B}>$ $S_{B A}$ and $u^{B}>V(B)$, where $V(B)$ is the coalitional value of agent $B$ in a single agent coalition.

The agents compare their maximum surpluses, and the one with the larger maximum surplus is stronger. The stronger agent can claim a part of the weaker agent's payoff, but this claim is limited by the individual rationality: $u^{B}>V(B)$. Therefore, agent $A$ cannot claim an amount that would leave agent $B$ with $V(B)$ or less. If two agents cannot outweight one another, they are in equilibrium: $A$ and $B$ are in equilibrium if one of the following conditions is satisfied: $1 . S_{A B}=S_{B A} ; 2 . S_{A B}>$ $S_{B A}$ and $u^{B}=V(B) ; 3 . S_{A B}<S_{B A}$ and $u^{A}=V(A)$.

Note that equilibrium is defined only for pairs of distinct agents who are members of the same coalition.

Using the concept of equilibrium, the kernel can be defined as the set of all coalitional configurations (and its associated payoffs) such that every pair of agents within the same coalition are in equilibrium. A coalitional configuration (and payoff distribution) of this type is also called kernel stable (K-stable). Furthermore, the kernel always exists for any coalitional configuration. However, checking the stability does not direct the agents to a specific coalitional configuration. The following example from [17] illustrates a simple kernel configuration.

Consider a game given by the characteristic functions (the payoff that agents or coalitions or agents can obtain by themselves):

$v(A B)=90 ; \quad v(A C)=80 ; \quad v(B C)=70 ; \quad v(N)=105$

where there are three players: $A, B$ and $C$, and $N$ is the grand coalition formed by all three of them. Suppose players $A$ and $C$ are considering their coalition such that their payoff configuration is:

$$
(\mathrm{x} ; \mathcal{S})=(45,0,35 ; A C, B)
$$

${ }^{1}$ See [16] for details. 
where $\mathrm{x}$ is the payoff of each player and $S$ the coalition configuration. There are two coalitions that include $A$ but exclude $C$, namely coalitions $A$ and $A B$. The excess of coalition A with respect to $(\mathbf{x} ; \mathcal{S})$ is $v(A)-x_{A}=0-45=-45$, while the excess of coalition $A B$ is $v(A B)-x_{A}-x_{B}=90-45-0=45$. The maximum surplus of player $A$ over player $C$ is therefore $s_{A C}=\max (-45,45)=45$. Similarly, $s_{C A}=35$. Since $45>35$ and $x_{C}>0=v(C)$, player $A$ outweights player $C$ with respect to $(\mathrm{x} ; \mathcal{S})$ and the payoff configuration is not in the kernel of the game.

Now consider another payoff configuration for the same game, this time one with the grand coalition, or

$$
(\mathrm{x} ; \mathcal{S})=(50,30,25 ; A B C) .
$$

The maximum surplus of player $B$ over player $C$ is

$$
\begin{aligned}
s_{B C} & =\max \left(v(B)-x_{B}, v(A B)-x_{A}-x_{B}\right) \\
& =\max (-30,10)=10
\end{aligned}
$$

The converse maximum surplus is $s_{C B}=5$. Since $10>$ 5 and $\mathrm{x}_{c}>0$, player $B$ outweights player $C$ with respect to $(\mathrm{x} ; \mathcal{S})$, and the payoff configuration is not in the kernel. But consider now the payoff configuration

$$
(\mathrm{x} ; \mathcal{S})=(45,35,25 ; A B C) .
$$

Here, we find that $s_{A B}=s_{A C}=s_{C A}=s_{B C}=s_{C B}=10$. Therefore, all players are in equilibrium, and the payoff configuration is a point of the kernel.

In the transmission planning problem considered as a cooperative game, the steps that are taken to build coalitions and to allocate costs are based on the Kernel-oriented Coalition Algorithm (KCA) developed by Klusch and Shehory [18], [19]. This is a new formulation of the same problem as seen in [14] where a Bilateral Shapley Value (BSV) algorithm was presented.

As said above, the KCA is a decentralized, negotiation-oriented coalition algorithm which determines a kernel-stable payment. The coalition negotiation is round-based, which means that all agents are synchronized at specified points in the negotiations. At the end of each round a new coalition is formed out of two old coalitions or there are no accepted coalition proposals and the algorithm stops with a kernel-stable payment configuration. For more details on how the agents are defined at the beginning of the game see [14].

Each round of the KCA can be divided in three phases:

\section{A. Calculating and Sending Coalition Offers}

Initially, all players are single coalitions. The coalition chooses the strongest (in the kernel sense) representative to calculate coalition offers. Her task is to calculate the new payoff vector of every joint coalition that may be possible to create. Then, a K-stable payment configuration is calculated for each coalition structure that can be formed out of the old structure by joining the own coalition with one in the ranking list. Later, the representative sends a proposal to every promising coalition.

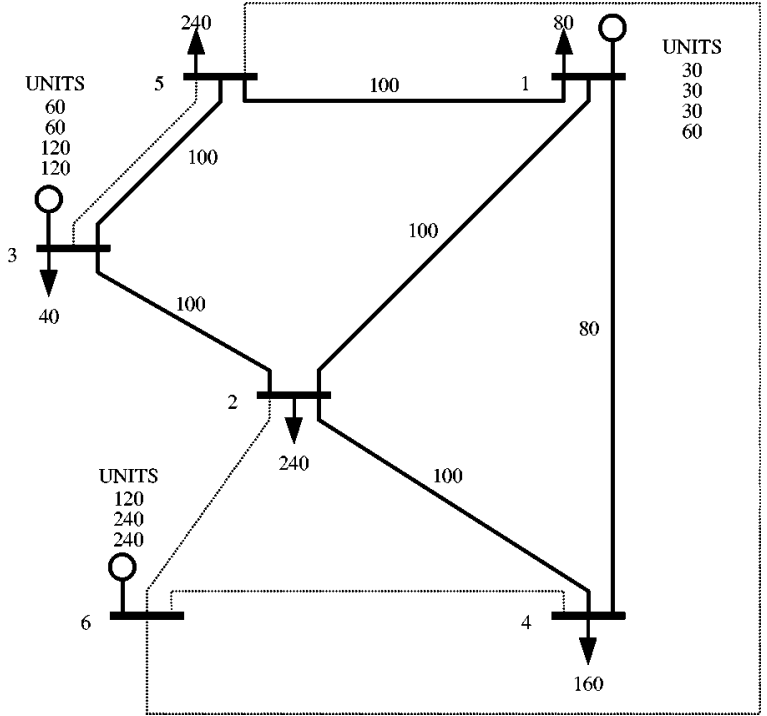

Fig. 1. 6 bus Garver test system.

\section{B. Coalition Formation}

If the payment to all members of the joint coalition is greater than their current payoffs in their original coalitions, and if the received offer is the first in the ranking list, then the proposal is accepted. This local decision is broadcasted to all current coalitions.

\section{Cost Allocation and Stopping Rules}

Cost are allocated at every iteration using the K-stable concept, and when the coalition formation process ends, the allocated cost is the last one calculated. Negotiation continues until all proposals of all coalition entities of the current coalition structure are rejected, or a grand coalition has been already formed, or a pre-defined time period has been exceeded.

\section{NumericAl Tests}

To test the KCA model we have run simulations with two different transmission expansion planning examples. A dual software platform has been implemented with that purpose. The first component simulates the coalition formation process and the second one the cost allocation procedure. The software implementation is shown in great detail in [20], [21].

\section{A. 6 Bus Garver Test System KCA Solution}

The first example is the classical 6 bus example from Garver [1], as shown in Fig. 1. Cost data and coalitional values are presented in Tables I and II, respectively. Note that the costs are equivalent to negative values in Table II.

The kernel algorithm rewards strong agents from the beginning, and this is the case of agent 6 . Bus 6 is constantly needed to supply the load for most of the other buses and it is rewarded by the kernel method because agent 6 excesses with respect to possible coalition partners are high.

Following the kernel-stable algorithm formulated above, the coalition formation process is as follows: $[1,\{2-6\}, 3,4,5] \rightarrow$ $[\{1-2-6\}, 3,4,5] \rightarrow[\{1-2-4-6\}, 3,5] \rightarrow[\{1-2-3-4-6\}, 5] \rightarrow$ 
TABLE I

6 Bus Garver Test System Data

\begin{tabular}{c|c|c|c}
\hline \hline $\begin{array}{c}\text { Bus } \\
\text { from/to }\end{array}$ & $\begin{array}{c}\text { Cost } \\
\text { (Units) }\end{array}$ & $\begin{array}{c}\text { Susceptance } \\
(1 / \Omega)\end{array}$ & $\begin{array}{c}\text { Capacity } \\
(\mathrm{MW})\end{array}$ \\
\hline $1 / 2$ & 40 & 2.50 & 100 \\
\hline $1 / 4$ & 60 & 1.67 & 80 \\
\hline $1 / 5$ & 20 & 5.00 & 100 \\
\hline $2 / 3$ & 20 & 5.00 & 100 \\
\hline $2 / 4$ & 40 & 2.50 & 100 \\
\hline $2 / 6$ & 30 & 3.33 & 100 \\
\hline $3 / 5$ & 20 & 5.00 & 100 \\
\hline $4 / 6$ & 30 & 3.33 & 100 \\
\hline $5 / 6$ & 61 & 1.64 & 78 \\
\hline \hline
\end{tabular}

TABLE II

COALITION EXPANSION VALUES FOR THE 6 BUS SySTEM

\begin{tabular}{|c|c|c|c|}
\hline Coalition & Value & Coalition & Volue \\
\hline 1 & 0 & $\{2,5,6\}$ & -334 \\
\hline 2 & -90 & $\{3,5,6\}$ & -81 \\
\hline 3 & 0 & $\{4,5,6\}$ & -304 \\
\hline 4 & -60 & {$[1,2,3,6]$} & -30 \\
\hline 5 & -40 & {$[1,2,4,6]$} & -120 \\
\hline 6 & -60 & $(1,2,5,6]$ & -273 \\
\hline$[2,6]$ & 90 & {$[1,4,5,6]$} & -243 \\
\hline$(3,5)$ & 40 & $(2,3,4,6]$ & -120 \\
\hline$(4,6)$ & -60 & {$[2,3,5,6]$} & -100 \\
\hline$(5,6]$ & -244 & $(3,4,5,6]$ & -141 \\
\hline$\{1,2,6\}$ & -60 & $\mid 1,2,3,4,6\}$ & -90 \\
\hline$\{1,3,5\}$ & -20 & $\{1,2,3,5,6\}$ & -80 \\
\hline $\mid 1,4,6\}$ & -60 & $\{1,2,4,5,6\}$ & -272 \\
\hline$\{1,5,6\}$ & -183 & $\{1,3,4,5,6\}$ & -70 \\
\hline $\mid 2,3,6\}$ & -60 & $\{2,3,4,5,6\}$ & -160 \\
\hline$|2,4,6|$ & -150 & {$[1,2,3,4,5,6]$} & -130 \\
\hline
\end{tabular}

[\{1-2-3-4-5-6\}]. The final cost allocation is: $(16.25,-76.25$, $16.25,-60,-40,13.75)$.

\section{B. 6 Bus Garver Test System BSV Solution}

Previous work based on Bilateral Shapley Values [13], [14] showed several possible grand coalition cost allocation solutions:

From Table III, it is observable that the BSV method does not favor big players as much as the kernel. More fairness in the cost allocation can be expected from BSV's, acting in a Shapley-like fashion. This fact is observed in the allocation of cost to buses 4, 5 and 6 , where Table III shows that bus 6 must always pay in contrast to the kernel solution where the other agents subsidize bus 6 (the strongest) increasing their own payments.

\section{6 Bus Garver Test System Sunk Costs Allocation}

A variation of the six bus example is the cost allocation of all lines: existing and possible new ones, assuming a total expansion cost of 130 monetary units, the grand coalition scheme. Table IV shows the new costs. Kernel allocation results are as follows: $(0,-90,0,-60,-40,0)$. The simulation shows that only buses 2 and 6 form a coalition and the process ends after 1 step. The reason is that sunk costs exceed the cost limits that
TABLE III

6 Bus Bilateral Shapley Value Algorithm Results

\begin{tabular}{c} 
BSV COST ALLOCATION \\
$\frac{[22.5,-78.75,7.5,-24.375,-32.5,-24.375]}{[[20,-80,10,-25,-30,-25]}$ \\
\hline$[11.25,-75,11.25,-24.375,-28.75,-24.375]$ \\
\hline$[16.25,-75,8.125,-23.75,-31.875,-23.75]$ \\
\hline$[21.25,-49.375,6.25,-55,-33.75,-19.375]$ \\
\hline$\frac{[15,-78.75,22.5,-24.375,-40,-24.375]}{[15,-48.75,22.5,-60,-40,-18.75]}$ \\
\hline$[12.5,-49.375,10.625,-55,-29.375,-19.375]$ \\
\hline
\end{tabular}

TABLE IV

Sunk Costs For the 6 Bus Garver Test System

\begin{tabular}{|c|c|c|c|}
\hline Condition & Value & Cocalition & Value \\
\hline 1 & 0 & $\{2,5,6\}$ & -334 \\
\hline 2 & -90 & $\mid 3,5,6\}$ & -101 \\
\hline 3 & 0 & $\mid 4,5,6\}$ & -304 \\
\hline 4 & -60 & {$[1,2,3,6]$} & -90 \\
\hline 5 & -40 & {$[1,2,4,6]$} & -260 \\
\hline 6 & -60 & $(1,2,5,6)$ & -333 \\
\hline$(2,6)$ & 90 & $(1,4,5,6]$ & -323 \\
\hline$[3,5]$ & -60 & $(2,3,4,6]$ & -180 \\
\hline$(4,6]$ & -60 & {$[2,3,5,6]$} & -140 \\
\hline$(5,6]$ & -244 & $(3,4,5,6]$ & -161 \\
\hline $\mid 1,2,6\}$ & -100 & $\mid 1,2,3,4,6\}$ & -250 \\
\hline$\{1,3,5\}$ & -60 & $\{1,2,3,5,6\}$ & -180 \\
\hline $\mid 1,4,6\}$ & -120 & $\{1,2,4,5,6\}$ & -432 \\
\hline $\mid 1,5,6\}$ & -203 & $\{1,3,4,5,6\}$ & -170 \\
\hline $\mid 2,3,6\}$ & -120 & $\{2,3,4,5,6\}$ & -240 \\
\hline$|2,4,6|$ & -190 & {$[1,2,3,4,5,6]$} & .330 \\
\hline
\end{tabular}

make buses attractive to possible partners. Therefore, sunk costs cannot be calculated using the algorithm proposed in Section IV. BSV's would lead to similar conclusions.

\section{IEEE 24 Bus RTS Example}

The final example is taken from the IEEE 24 bus Reliability Test System (RTS), as shown in Fig. 2 and Tables V-VII. Note that Fig. 2 presents the IEEE 24 bus system divided in four initial coalitions that fulfill the self-sufficiency axioms described in [14]. Table $\mathrm{V}$ shows the possible new lines in shadowed grey color and total generation $(G)$ and demand $(D)$ for each coalition are also shown in Fig. 2. It is also assumed that line thermal limits are equal to $3333 \mathrm{MW}$ for all lines.

The KCA produces the coalition formation process and final cost allocation as presented in Fig. 3.

These results indicate that self-sufficient players, like coalition 4, with no need to expand their own system, will benefit from other player's gradual expansion plans. In particular, total system reliability is greatly enhanced with player 4 and that is why player 4 gets a positive reward whilst players 1,2 , and 3 have to pay for expansion, although much less than in isolation.

\section{CONCLUSION}

This paper has shown a new decentralized coalition formation and cost allocation procedure based on a kernel approach. 


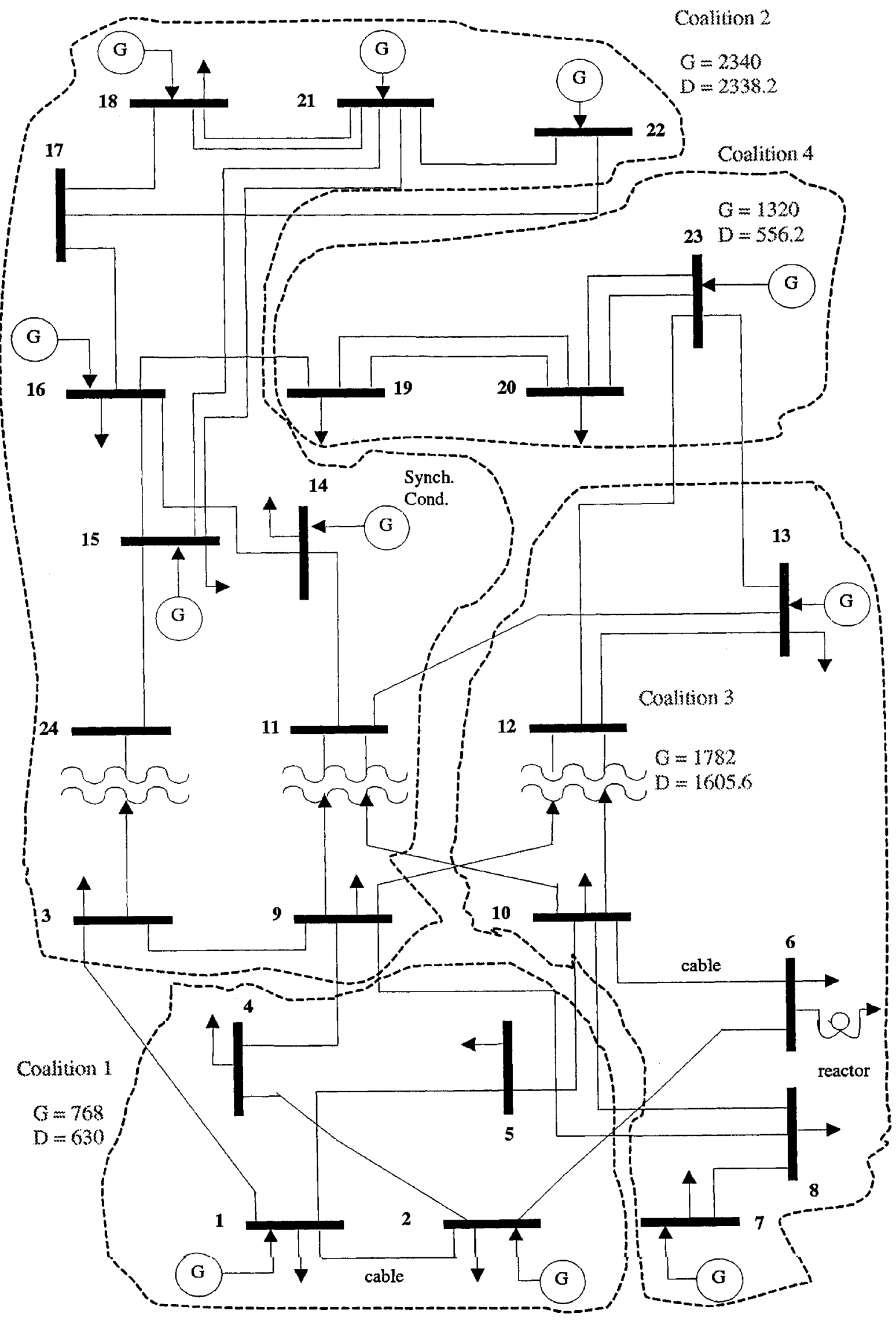

Fig. 2. IEEE 24 bus RTS example. 
TABLE $\mathrm{V}$

IEEE 24 BUS RTS LINE CHARACTERISTICS

\begin{tabular}{c|c|c|c}
\hline LINE & REACTANCE & LINE & REACTANCE \\
\hline$(1,2)$ & 7.04 & $(10,12)$ & 42.47 \\
\hline$(1,3)$ & 106.92 & $(11,13)$ & 24.10 \\
\hline$(1,5)$ & 42.78 & $(11,14)$ & 21.16 \\
\hline$(2,4)$ & 64.14 & $(12,13)$ & 24.10 \\
\hline$(2,6)$ & 97.20 & $(12,23)$ & 48.90 \\
\hline$(3,9)$ & 60.24 & $(13,23)$ & 43.79 \\
\hline$(3,24)$ & 42.47 & $(14,16)$ & 19.70 \\
\hline$(4,9)$ & 52.50 & $(15,16)$ & 8.76 \\
\hline$(5,10)$ & 44,70 & $24(15,21)$ & 24.81 \\
\hline$(6,8)$ & 31.08 & $(15,24)$ & 26.27 \\
\hline$(6,10)$ & 30.63 & $(16,17)$ & 13.11 \\
\hline $2^{*}(7,8)$ & 31.08 & $(16,19)$ & 11.70 \\
\hline$(8,9)$ & 83.58 & $(17,18)$ & 7.29 \\
\hline$(8,10)$ & 83.58 & $(17,22)$ & 53.31 \\
\hline$(9,11)$ & 42.47 & $2^{*}(18,21)$ & 13.11 \\
\hline$(9,12)$ & 42.47 & $2^{*}(19,20)$ & 20.05 \\
\hline$(10,11)$ & 42.47 & $2^{*}(20,23)$ & 10.93 \\
\hline & & $(21,22)$ & 34.32 \\
\hline
\end{tabular}

TABLE VI

IEEE 24 BUS RTS NODE CHARACTERISTICS

\begin{tabular}{c|c|c} 
NODE & $\begin{array}{c}\text { PRODUCTION } \\
(\text { MW })\end{array}$ & $\begin{array}{c}\text { DEMAND } \\
\text { (MW) }\end{array}$ \\
\hline 1 & 384 & 194.4 \\
\hline 2 & 384 & 174.6 \\
\hline 3 & 0 & 324 \\
\hline 4 & 0 & 133.2 \\
\hline 5 & 0 & 127.8 \\
\hline 6 & 0 & 244.8 \\
\hline 7 & 600 & 225 \\
\hline 8 & 0 & 307.8 \\
\hline 9 & 0 & 315 \\
\hline 10 & 0 & 351 \\
\hline 11 & 0 & 0 \\
\hline 12 & 0 & 0 \\
\hline 13 & 1182 & 477 \\
\hline 14 & 0 & 349.2 \\
\hline 15 & 430 & 570.6 \\
\hline 16 & 310 & 180 \\
\hline 17 & 0 & 0 \\
\hline 18 & 800 & 599.4 \\
\hline 19 & 0 & 325.8 \\
\hline 20 & 0 & 230.4 \\
\hline 21 & 800 & 0 \\
\hline 22 & 0 & 0 \\
\hline 23 & 1320 & 0 \\
\hline 24 & 0 & 0 \\
\hline & &
\end{tabular}

It has been tested with a simple 6 bus problem and a more realistic IEEE 24 bus RTS example with good results. However, the procedure has not solved the allocation of sunk costs; it will be subject of further research.

The main advantages of the KCA approach have been:

- Higher rewards to stronger players as compared to a BSV approach: agents that do not need to build new lines are always benefitted.
TABLE VII

VALUES FOR THE IEEE 24 BUS RTS EXAMPLE

\begin{tabular}{c|c|c}
\hline \hline COALITION & LINES & VALUE \\
\hline 1 & $1-2,2-4$ & -71.8 \\
\hline 2 & $9-11,11-14$ & -63.63 \\
\hline 3 & $8-10$ & -83.58 \\
\hline 4 & & 0 \\
\hline$\{1,2\}$ & $1-2,2-4,9-11,11-14$ & -134.81 \\
\hline$\{1,3\}$ & $1-2,2-4,8-10$ & -154.76 \\
\hline$\{2,3\}$ & $9-11,11-14$ & -63.63 \\
\hline$\{2,4\}$ & $9-11,11-14$ & -63.63 \\
\hline$\{3,4\}$ & $8-10$ & -83.58 \\
\hline$\{1,2,3\}$ & $1-2,9-11,11-14$ & -70.67 \\
\hline$\{1,2,4\}$ & $1-2,2-4,9-11,11-14$ & -134.81 \\
\hline$\{2,3,4\}$ & $3-9$ & -60.24 \\
\hline$\{1,2,3,4\}$ & $1-2,9-11$ & -49.51 \\
\hline \hline
\end{tabular}

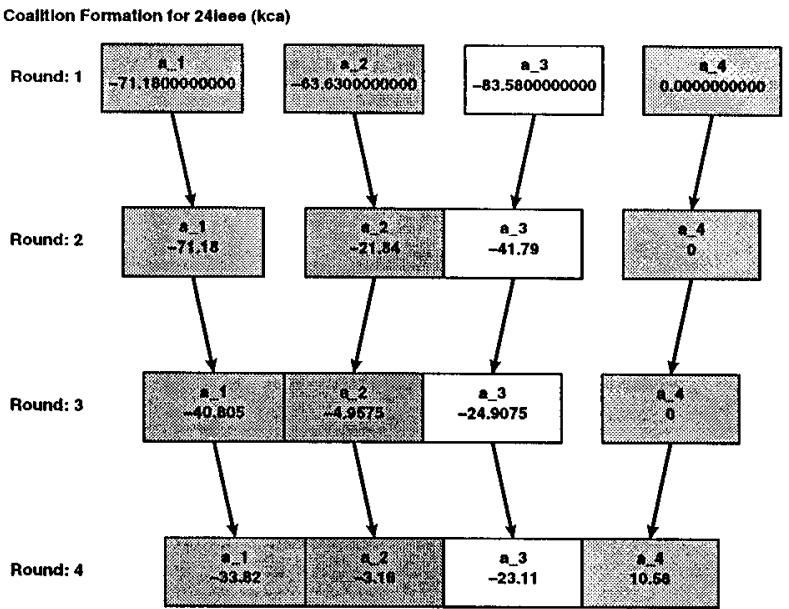

Fig. 3. IEEE 24 bus RTS example KCA results.

- Uniqueness of the solution to the transmission expansion problem, in contrast to a BSV approach.

\section{ACKNOWLEDGMENT}

The authors want to express their gratitude to J. Zolezzi Cid from the Pontificia Universidad Católica de Chile for his fruitful suggestions and corrections.

\section{REFERENCES}

[1] L. L. Garver, "Transmission Net estimation using linear programming," IEEE Trans. on Power Apparatus \& Systems, vol. PAS-89, no. 7, pp. 1688-1697, Sept./Oct. 1970.

[2] R. Romero and A. Monticelli, "A hierarchical decomposition approach for transmission network expansion planning," IEEE Trans. on Power Systems, vol. 9, no. 1, pp. 373-380, Feb. 1994.

[3] R. Romero and A. Monticelli, "A zero-one implicit enumeration method for optimizing investments in transmission expansion planning," IEEE Trans. on Power Systems, vol. 9, no. 3, pp. 1385-1391, August 1994.

[4] G. C. Oliveira, A. P. C. Costa, and S. Binato, "Large scale transmission network planning using optimization and heuristic techniques," IEEE Trans. on Power Systems, vol. 10, no. 4, pp. 1828-1833, Nov. 1995.

[5] R. Romero, R. A. Gallego, and A. Monticelli, "Transmission system expansion planning by simulated annealing," IEEE Trans. on Power Systems, vol. 11, no. 1, pp. 364-369, Feb. 1996. 
[6] K. Yoshimoto, K. Yasuda, and R. Yokohama, "Transmission expansion planning using neuro-computing hybridized with genetic algorithm," in Proceedings of the 1995 International Conference on Evolutionary Computing, ICEC'95, Perth, Australia, Nov./Dec. 1995, pp. 126-131.

[7] H. Rudnick, R. Palma, E. Cura, and C. Silva, "Economically adapted transmission systems in open access schemes-Application of genetic algorithms," IEEE Trans. on Power Systems, vol. 11, no. 3, pp. 1427-1440, Aug. 1996.

[8] R. Baldick and E. Kahn, "Transmission planning issues in a competitive economic environment," IEEE Trans. on Power Systems, vol. 8, no. 4, pp. 1497-1503, Nov. 1993

[9] E. L. Silva, S. E. C. Mesa, and M. Morozowski, "Transmission access pricing to wheeling transactions: A realiability based approach," IEEE Trans. on Power Systems, vol. 13, no. 4, pp. 1481-1486, Nov. 1998.

[10] J. W. Marangon Lima and E. J. de Oliveira, "The long- term impact of transmission pricing," IEEE Trans. on Power Systems, vol. 13, no. 4, pp. 1514-1520, Nov. 1998.

[11] M. Soto and H. Rudnick, "Generation and transmission contributions to system reliability in open access schemes," in Proceedings of the PES Winter Meeting 1999, pp. 416-421.

[12] D. Gately, "Sharing the gains from regional cooperation: A game theoretic application to planning investment in electric power," International Economic Review, vol. 15, no. 1, pp. 195-208, Feb. 1974.

[13] J. Contreras, "A Cooperative Game Theory Approach to Transmission Planning in Power Systems," Ph.D. dissertation, Department of Electrical Engineering and Computer Sciences, University of California at Berkeley, May 1997.

[14] J. Contreras and F. F. Wu, "Coalition formation in transmission expansion planning," IEEE Trans. on Power Systems, to be published.

[15] X. Wang and J. R. McDonald, Modern Power System Planning. Cambridge, U.K.: McGraw-Hill, 1994.

[16] R. Davis and D. Maschler, "The kernel of a cooperative game," Naval Research Logistics Quarterly 12, pp. 223-259, 1965.

[17] J. P. Kahan and A. Rapoport, Theories of Coalition Formation. London, U.K.: Lawrence Erlbaum Associates, Publishers, 1984
[18] M. Klusch and O. Shehory, "A polynomial kernel-oriented coalition algorithm for rational information agents," in Proceedings of the 2nd International Conference on Multi-Agent Systems. Kyoto, Japan, Dec. 9-13, 1996.

[19] O. Shehory and S. Kraus, "A kernel-oriented model for coalition formation in general environments: Implementation and results," in Proceedings of AAAI-96, Portland, OR, 1996.

[20] J. Contreras, M. Klusch, T. Vielhak, J. Yen, and F. F. Wu, "Multi-Agent coalition formation in transmission planning: Bilateral shapley value and kernel approaches," in Proceedings of the 13th Power Systems Computation Conference PSCC'99, Trondheim, Norway, June and July 28th and 2nd, 1999.

[21] "COALA and COPOP projects homepages,", www.informatik.tu-chemnitz.de/ klusch/coala.html and www.eecs.berkeley.edu/ javier.

Javier Contreras (M'98) was born in Zaragoza, Spain, in 1965. He received his B.S. in electrical engineering from the University of Zaragoza, Spain, his M.S. from the University of Southern California, and his Ph.D. from the University of California, Berkeley in 1989, 1992, and 1997, respectively. He was a Researcher at the Instituto de Investigación Tecnológica, Escuela Técnica Superior de Ingeniería (ICAI), Universidad Pontificia Comillas, in Madrid, Spain, from 1997 to 1998. He is currently an Assistant Professor at the E.T.S. de Ingenieros Industriales, Universidad de Castilla - La Mancha, in Ciudad Real, Spain.

Felix F. Wu received his B.S. from National Taiwan University, his M.S. form the University of Pittsburg, and his Ph.D. form the University of California, Berkeley. He worked for Pacific Gas and Electric as a Computer Applications Engineer in 1976-77. Dr. Wu was elected a Fellow of the IEEE in 1989. He held a TEPCO (Tokyo Electric Power Company) Professorship at the University of Tokyo in 1991. He has been a Professor of Electrical Engineering and Computer Sciences at the UC Berkeley; presently he is a Professor of Electrical Engineering at the University of Hong Kong, HKSAR. 